\title{
Violencia en la red social: una indagación de expresiones online en adolescentes de sectores populares marginalizados del Área Metropolitana de Buenos Aires
}

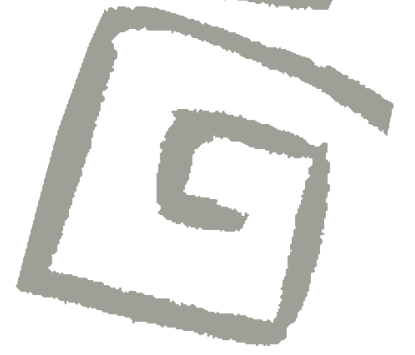

\author{
Violence in social networks: an exploration of the \\ online expressions of teens from marginalized areas of \\ Greater Buenos Aires
}

Joaquín Walter Linne', María Florencia Angilletta²

${ }^{1}$ Doctor en Ciencias Sociales. Investigador Asistente, Consejo Nacional de Investigaciones Científicas y Técnicas (CONICET) con sede en el Instituto de Investigaciones Gino Germani, Facultad de Sociología, Universidad de Buenos Aires. Profesor Adjunto, Universidad Nacional de Lanús, Buenos Aires, Argentina. $\square$ (iD

${ }^{2}$ Licenciada en Letras. Becaria doctoral, Consejo Nacional de Investigaciones Científicas y Técnicas (CONICET) con sede en el Instituto de Filología y Literaturas Hispánicas, Facultad de Filosofía y Letras, Universidad de Buenos Aires, Buenos Aires, Argentina. $\square$ (iD)
RESUMEN Este trabajo explora las expresiones online de violencia ejercida o padecida por las y los adolescentes de sectores populares marginalizados del Área Metropolitana de Buenos Aires. Desde una metodología cualitativa, se indagan cuatro fenómenos específicos: las amenazas, los "bondis", el cyberbullying y los duelos, para lo cual se realizaron veinte entrevistas en profundidad y 3.000 observaciones virtuales de perfiles de la red social Facebook entre 2013 y 2014. Entre los principales resultados, se observa que la mayoría de las expresiones de violencia se enmarcan en una dinámica offline-online. Asimismo, se ofrece evidencia empírica a partir de la cual es posible afirmar que las expresiones de violencia de estos adolescentes se despliegan en torno a la cultura del "aguante". El artículo se pregunta si en la plataforma icónica del "me gusta" estas expresiones resultan implícitamente funcionales a la red social o si, por el contrario, permiten desplazamientos y reapropiaciones significativas de los usuarios. En definitiva, se abren nuevos interrogantes acerca de la utilización de estas herramientas por parte de adolescentes de sectores populares marginalizados y se propone complejizar los enfoques en torno a estos fenómenos.

PALABRAS CLAVES Adolescentes; Redes Sociales; Violencia; Población Suburbana; Argentina.

\begin{abstract}
This paper explores the online expressions of violence perpetrated or experienced by adolescents from marginalized areas of Greater Buenos Aires, Argentina. Using a qualitative methodology, four specific events were examined: threats, "bondis" [fights], cyberbullying and displays of mourning. To do so, 20 in-depth interviews and 3,000 virtual observations of profiles in the social network Facebook were carried out. Among the main results, it was seen that most expressions of violence are part of an offline-online dynamic. Empirical evidence is also offered based upon which it can be affirmed that the expressions of violence of these teenagers are developed around the culture of "aguante" [fierce loyalty]. The article ponders the extent to which, in the iconic platform of the option "like," these expressions are implicitly functional to the social network or, to the contrary, or whether they allow displacements and significant reappropriations on the part of users. New questions arise about the use of these tools by adolescents from marginalized areas and the need for more complex approaches to examine these phenomena.
\end{abstract}

KEY WORDS Adolescents; Social Networking; Violence; Suburban Population; Argentina. 


\section{INTRODUCCIÓN Y PLANTEO DEL PROBLEMA}

Tal como puede relevarse en el estado de la cuestión, la violencia no admite una definición única. En efecto, los cambios sociohistóricos muestran las disputas en las sociedades por delimitar los fenómenos de la violencia, ya sea como condiciones materiales, prácticas simbólicas, uso de la fuerza física, entre otros. Sin desconocer la amplitud de estas discusiones, puede plantearse aquí una definición biopolítica de la violencia como una práctica productiva -en el sentido de generadora de prácticas- y no simplemente restrictiva. Foucault ${ }^{(1,2)}$ sostiene que en las sociedades modernas las estrategias negativas y tradicionalmente coercitivas se integran en una red más compleja del dispositivo que denomina "biopolítico" para la fabricación de "cuerpos dóciles". El "biopoder" ya no se concentra solo en la fuerza del "castigo", sino que implica diversos sistemas de producción de "vigilancia" sobre los cuerpos. En su analítica del poder y la violencia, el autor señala que se trata de un poder que opera por producción, intensificación y administración: "Ya no se trata de hacer jugar la muerte en el campo de la soberanía, sino de distribuir lo viviente en un dominio de valor y de utilidad"(2 p.136).

Desde su propia genealogía, la Modernidad se encuentra atravesada por distintas formas de violencia. Las complejas relaciones entre Estado, ley y violencia construyen una serie de modulaciones que permiten resignificar la propia concepción de Modernidad en el marco de las revoluciones burguesas. Al respecto, resulta sintomático que uno de los hitos fundacionales de la edad contemporánea sea la Revolución Francesa, cuyo símbolo apelaba a la literalidad de "cortarle la cabeza a la monarquía". Desde una óptica novedosa, Benjamin ${ }^{(3)}$ propuso revisar los conflictivos lazos entre violencia y orden democrático. Asimismo, como se ha señalado, Foucault ${ }^{(1)}$ ha estudiado los vínculos entre violencia y poder, mostrando que las relaciones no pueden ser pensadas solo de un modo dual y jerárquico, sino más bien como nodos capilares y multifacéticos, en lo que denomina una "micropolítica".

Ya en las coordenadas del siglo XX, las experiencias límite que marcan las dos guerras mundiales y los sucesivos conflictos bélicos concitan nuevas reflexiones sobre la violencia. Autores como Sloterdijk ${ }^{(4)}$ y Ranciére(5) señalan esta "crisis del humanismo" y sus efectos en torno a problemáticas globales de violencia como el terrorismo, la pobreza urbana, la crisis de los refugiados y la violencia de género. Asimismo, tanto Isla y Míguez ${ }^{(6)}$ como Spinelli et al. ${ }^{(7)}$ y Tonkonoff ${ }^{(8)}$ coinciden en que la violencia es una construcción social e histórica cuyas prácticas se transforman según las negociaciones y consensos establecidos en cada sociedad. Este recorrido, sin dudas nada exhaustivo, permite al menos señalar que toda reflexión sobre la violencia, para no resultar una lectura estigmatizante sobre un sector social, debe pensarse necesariamente en el campo más amplio de la vida democrática, institucional y jurídica.

Uno de los fenómenos contemporáneos que más afecta a la población mundial es la violencia urbana, definida aquí como el conjunto específico de acciones violentas que se producen en las ciudades y que altera su entramado social ${ }^{(9)}$. Algunos ejemplos de violencia característicos de megalópolis, ciudades capitales y metrópolis son los robos y asesinatos, así como también las peleas callejeras, las infracciones, las evasiones impositivas, los accidentes de tránsito y los disturbios en canchas de fútbol.

En particular, los sectores socialmente vulnerables ${ }^{(10)}$ se ven afectados por formas de violencia asociadas a las dificultades socioeconómicas, como la falta de empleo estable y la carencia de acceso a servicios sociales básicos. En Argentina, los barrios con estas características suelen denominarse "villas de emergencia", "villas miseria" o "asentamientos urbanos precarios". Estos barrios también son definidos como "márgenes urbanos" $^{\prime \prime(10,11)}$, en el sentido de que son territorios segregados de las zonas más desarroIladas. Se trata, en definitiva, de áreas que 
suelen encontrarse apartadas y con menos facilidad para acceder a servicios sociales, de salud y medios de transporte ${ }^{(12)}$. Wacquant ${ }^{(13)}$, quien investiga el fenómeno de estratificación social que atañe a estos sectores, define como "procesos de guetificación urbana" a la aislación social progresiva que atraviesan distintos barrios en los que residen estas poblaciones.

Dentro del contexto nacional, Auyero, Burbano de Lara y Berti ${ }^{(14)}$, Di Leo ${ }^{(15)}$, Isla y Míguez $^{(6)}$, Kessler y Dimarco ${ }^{(11)}$, Spinelli et al. $^{(7)}$, y Tonkonoff( ${ }^{(8)}$ analizan las distintas formas de violencia desplegadas durante los últimos años en márgenes urbanos del Área Metropolitana de Buenos Aires (AMBA) ${ }^{[a]}$ : en primer lugar, una violencia interpersonal a raíz de enfrentamientos entre vecinos, familiares o pares; en segundo lugar, una violencia relacionada con actividades criminales, problemáticas sociales y la acción punitiva estatal. Estos autores señalan que la violencia suele ser una de las principales preocupaciones de los habitantes socialmente vulnerables del AMBA.

Si bien el estado de la cuestión ha estudiado las formas de violencia copresencial en sectores socialmente vulnerables, la incidencia de la masificación de las tecnologías de la información y la comunicación (TIC) en los jóvenes del AMBA requiere ampliar este análisis para incluir fenómenos emergentes, que son los indagados en este artículo. Morduchowicz $^{(16)}$ registra que la socialización de las y los adolescentes se despliega en un continuum offline-online. Del mismo modo, puede pensarse que la violencia que experimentan se desenvuelve en un contexto análogo. Así se entrelazan peleas, robos, estigmatización social, segregación escolar y violencia de género con fenómenos surgidos a partir de la masificación de las TIC.

Son significativos los estudios recientes sobre violencia en Latinoamérica ${ }^{(9,17,18)}$ y en Argentina ${ }^{(6,7,8,10,11,14,15,19)}$. En particular, se ha revisado la bibliografía que aborda las expresiones de violencia en "sitios de redes sociales", encontrando una escasez de estudios sobre adolescentes de sectores populares, dado que la mayoría de las investigaciones se circunscribe a jóvenes de sectores $\operatorname{medios}^{(20,21,22,23,24)}$. En esta línea, en pos de contribuir al conocimiento de esta temática tan compleja y contemporánea, el presente artículo se centra en las expresiones de violencia online que realizan poblaciones juveniles de sectores populares del AMBA en Facebook.

La adolescencia es una etapa central de configuración identitaria en la que resultan claves las prácticas exploratorias ${ }^{(25,26)}$. Con la masificación y adopción de las TIC en la vida cotidiana de una vasta mayoría de adolescentes, una parte significativa de esta configuración identitaria y de estas prácticas exploratorias suele estar asociada al perfil que los jóvenes construyen en Facebook ${ }^{(27)}$. Se lo elige entonces como plataforma de análisis porque se ha convertido en la mayor red social a nivel mundial y funciona como sinónimo de Internet para una gran parte de estos adolescentes, dado que allí despliegan la mayor parte de sus acciones de comunicación y entretenimiento online ${ }^{(28)}$. Por lo tanto, esta plataforma representa un ámbito privilegiado para indagar las prácticas de esta población.

A partir del trabajo de campo, se desarroIlaron dos líneas de investigación exploratoria sobre las expresiones de violencia que estos adolescentes despliegan en Facebook: una primera dimensión refiere a las prácticas violentas que realizan los usuarios en un circuito offline-online; una segunda dimensión alude a las prácticas que les permiten a los usuarios expresar y resignificar la violencia que padecen en el mismo circuito. En la primera línea, se incluyen las amenazas, los "bondis" y el cyberbullying. En la segunda, se indagan los duelos. Ambas dimensiones generan interrogantes acerca de si Facebook es un mero dispositivo reproductor de estos fenómenos o si les permite a estas poblaciones un empoderamiento frente a la violencia que padecen.

\section{MÉTODO}

Esta investigación, surgida a partir de un trabajo de mayor extensión ${ }^{(27)}$, es exploratoria 
y cualitativa. Se sustenta sobre la base de una muestra no intencional de adolescentes del AMBA, conformada a través del método "bola de nieve". Se realizaron veinte entrevistas en profundidad en aulas de colegios o centros de inclusión digital[b], que tuvieron una duración aproximada de una hora, y observaciones copresenciales que fueron realizadas en colegios, ciberlocutorios, vía pública y centros de inclusión digital.

El sujeto de investigación se construye en la intersección de dos variables: la edad y el sector social. Asimismo, se tomaron recaudos metodológicos para no homogeneizar al sujeto de estudio desde una mirada "androcéntrica"(29), sino indagar las distintas modulaciones que evidencian mujeres y varones. Con relación a la primera variable, dentro de la categoría juventud, que ha sido definida por distintos autores como una construcción social(26,30), este trabajo se concentra en las y los adolescentes entre 13 y 18 años de edad. En cuanto a la segunda variable, aquí se propone estudiar a las y los adolescentes de sectores socialmente vulnerables.

Distintos estudios ${ }^{(6,10,12,30)}$ coinciden, aun con sus matices, en definirlos como aquellas poblaciones que viven en condiciones socioeconómicas inestables y residen en barrios que carecen de servicios sociales básicos, lo que les produce cierto efecto de aislamiento social respecto de la metrópolis a la que pertenecen o se encuentran cercanos. Partiendo de la productividad analítica de estos trabajos, y de los datos del Instituto Nacional de Estadística y Censos (INDEC) ${ }^{(12)}$, aquí se propone definir a estos grupos sociales como sectores populares y subdividirlos entre integrados y marginalizados. Por un lado, resulta necesario realizar distinciones dentro de este grupo. Por otro lado, esta subcategorización reviste además la búsqueda de una mayor precisión sociológica para describir a estos grupos sociales e incrementar las posibilidades de transferencia. Lejos de pretender acrecentar la estigmatización sufrida por estos adolescentes, se procura contribuir al estado de la cuestión y proponer herramientas de investigación que resulten útiles para optimizar las políticas públicas orientadas a esta población.
En comparación con las tres décadas pasadas, si bien existe una mayor presencia estatal en asistencia socioeconómica y en transferencia de recursos tecnológicos a los sectores socialmente vulnerables, con iniciativas como la Asignación Universal por Hijo, el Programa Conectar Igualdad, el Programa Jóvenes con Más y Mejor Trabajo y el Plan Fines ${ }^{[c]}$, todavía quedan numerosos adolescentes de sectores populares que atraviesan condiciones de vida ante las cuales el Estado debe continuar generando respuestas. Los sectores populares integrados del AMBA, por su mayor nivel de participación en el sistema educativo, el mercado de trabajo formal y el sistema de salud, suelen ser los mayores beneficiarios del impacto de estas políticas públicas. En cambio, los sectores populares marginalizados mantienen un vínculo más intermitente y conflictivo con estas instituciones $y$, por lo tanto, pese a ser quienes más necesitan de la intervención directa del Estado, en numerosos casos atraviesan los efectos de las políticas públicas con mayor grado de ambivalencia. En efecto, las poblaciones aquí definidas como sectores populares marginalizados padecen condiciones vitales más extremas y conflictivas: poseen padres desempleados de larga duración o subempleados de baja calificación; residen en viviendas y barrios precarios; asisten al colegio con dificultades e intermitencias, o lo han abandonado por diferentes razones.

Uno de los grandes desafíos de la investigación en ciencias sociales es cómo acercarse a estas poblaciones vulnerables, que justamente tienen un contacto intermitente con las instituciones estatales. En este sentido, la elección de la red social Facebook contribuye a una estrategia metodológica que representa una vía posible de acceso al trabajo de campo con estos adolescentes. Una de las herramientas desarrolladas para llevar a cabo el trabajo fue la creación y mantenimiento de un perfil ad hoc en Facebook. Esta línea de observación fue construida con el fin de estudiar virtualmente a los adolescentes, según recomendaciones de distintas investigaciones que realizan etnografía digital para comprender a una población que considera 
central estos dispositivos ${ }^{(23,24,33,34)}$. Durante el trabajo docente ad honorem en un centro de inclusión social de la zona sur del AMBA, un colega y referente barrial comentó que utilizaba Facebook para "monitorear" a los adolescentes de la zona; esto resultó un recurso valioso para avanzar con la investigación, por lo que se creó un perfil con fines analíticos, a partir del cual se agregaron contactos en común que fuesen adolescentes de sectores populares. El perfil ad hoc se ha limitado a observar, comentar y colocar "me gusta" en algunas publicaciones.

A través del desarrollo de esta cuenta de Facebook, que poseía 3.000 contactos al momento del estudio, se exploraron los modos en que las y los adolescentes se autopresentan e interactúan entre sí, así como las estrategias y recursos que utilizan para diseñar y actualizar sus perfiles en la red social. Esta instancia experimental desarrollada entre 2013 y 2014 resultó una de las aristas más significativas del trabajo de campo, dado el profuso material de archivo recogido, catalogado y analizado. Para ordenar las imágenes y los textos personales recolectados, se crearon carpetas con distintos títulos, que después permitieron la proposición analítica de los fenómenos de violencia. Si bien se prosiguió activamente con varias instancias clásicas de la investigación, estos aspectos etnográficovirtuales brindaron elementos de análisis de la acción significativa de las y los adolescentes que no siempre podían encontrarse in situ. Esta catalogación permitió detectar, a lo largo de tres años de observaciones virtuales, la aparición recurrente de ciertas expresiones como "aguante", "bardeos" o "pararse de manos"[d].

Por cuestiones éticas, se pidió autorización a los entrevistados y no se guardó registro de datos personales. Se aclara que Facebook es un sitio de redes sociales público que posee condiciones y políticas similares a las de la mayoría de estos sitios: no exige restricciones para mayores de 13 años, dado que cualquier persona mayor de esa edad puede utilizarlo, y todos los usuarios firman un contrato con Facebook en el que aceptan que sus publicaciones estén online. Para preservar la identidad de las y los adolescentes, se colocaron etiquetas genéricas en cada extracto de entrevista citada. En los fragmentos de entrevistas y las transcripciones de las capturas de muros de Facebook, solo se señala si el usuario es varón o mujer y su edad.

\section{RESULTADOS}

\section{Amenazas}

Durante el trabajo de campo, se detectó que una de las expresiones de violencia online más recurrente entre las y los adolescentes de la muestra era la que implica la sugerencia, posibilidad o advertencia de una violencia futura. Este tipo de expresiones son aquí denominadas "amenazas". En muchos casos, estas prácticas pretenden generar miedo en el receptor e incluso disuadirlo de alguna acción concreta, de modo directo o indirecto. Aunque convencionalmente las amenazas tienen como fin advertir de modo violento a un sujeto para que realice o no una acción, en las prácticas observadas se evidencia que estas amenazas están dirigidas a un grupo no siempre especificado, y se concentran en que el perfil del usuario que amenaza genere respeto entre sus pares.

En este punto, es necesario retomar el concepto de cultura del "aguante"(35), central en los universos de sentido de las y los adolescentes de sectores populares marginalizados del AMBA. Esta categoría nativa refiere originalmente a ciertas asociaciones de sentido y prácticas vinculadas a la capacidad de soportar los momentos adversos del club de fútbol como, por ejemplo, los descensos de categoría y los enfrentamientos con otras "barras", así como peleas en el barrio y en distintas instituciones ${ }^{(36)}$. Esta cultura del "aguante" luego va adquiriendo otras modulaciones, asociadas a la cultura del "reviente" y a distintos modos de exteriorización de la masculinidad y del ser legítimo. Como señalan las investigaciones citadas, para los jóvenes de sectores populares marginalizados del AMBA "aguante implica fidelidad, sostén, permanencia" ${ }^{\prime(37 \text { p.283) }}$. En este sentido, como en 
ciertos casos, la amenaza, más que dirigirse a otro usuario en particular de la red social, se difumina hacia el anonimato, el efecto que produce puede pensarse en términos de "aguante", dado que el usuario exhibe su capacidad de resistencia, de hacerle frente a la adversidad, de no "arrugar" ${ }^{\text {[e] }}$ y "pararse de manos ante cualquiera".

Sos puro teclado gila yo no me cagó cuando quieras sale pelea, jaja pero entre nosotras ya nos peliamos una vez y llamaste ah todo tu mambo te ases a la piola xq conoses a los de celinna 'pompeya jaja me chupa un huevo eso el problema es con vos yo se que me tenes miedo [emoticones] así que cuando quieras un mano ah mano rata , esos barrentines guardatelo para la verduleria a mi nadie me la boconia menos vos así que voffi [emoticón]

Sentiré $[P]$

(Publicación en Facebook, usuario del AMBA)

La transcripción corresponde a la publicación de una adolescente (16 años) que amenaza a otra y le aclara que ella no tiene miedo a enfrentarse físicamente. A partir de una serie de referencias que pueden encontrarse en esta publicación, se advierten distintas intersecciones entre los ámbitos offline y online. En primer término, quien amenaza alude a una historia en común con la amenazada: "entre nosotras ya nos peliamos una vez". En segundo término, al mencionar "celinna "pompeya" da cuenta de una referencia territorial relacionada con sus barrios de pertenencia identitaria, que implica también una forma de "aguante". Por último, con la expresión "mano a mano" indica su disponibilidad para un enfrentamiento físico. La amenaza, en tanto implica la eventual aplicación de una violencia futura en un ámbito copresencial, muestra que estas expresiones suelen ser parte de un continuum offline-online. La amenaza es online, pero la clave de lectura remite a un espacio offline.

Otra práctica habitual entre las y los adolescentes de la muestra consiste en colocar en su perfil o estado de Facebook fragmentos de letras de canciones que para ellos tematizan su situación vital, que a su vez pueden funcionar como amenazas. Esto se evidencia en que varios de los nombres de bandas de cumbia $^{[f]}$ y letras de canciones mencionadas aluden a la persecución policial, el encierro carcelario y el tráfico o consumo de drogas.

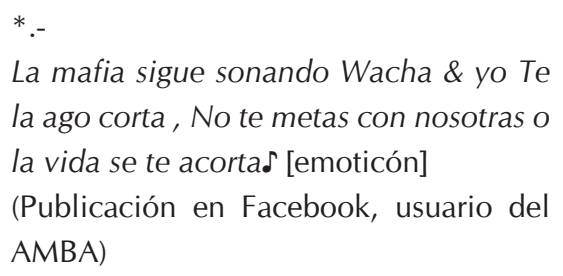

En este ejemplo se lee lo que escribe una adolescente (16 años). Míguez ${ }^{(38)}$, Semán y $\mathrm{Vila}^{(39)}$, y Silba ${ }^{(40)}$ coinciden en que las letras de cumbia villera generan identificación entre estos grupos porque presentan narraciones de la vida cotidiana en torno a la inseguridad de sus barrios y a ciertas actividades como el consumo de drogas, episodios violentos y enfrentamientos con la policía, que muchas veces van desde el careo y la requisa arbitraria hasta la represión y el "gatillo fácil". En este ejemplo en particular, se observa cómo la adolescente utiliza el fragmento de la canción para reforzar su modo de ser legítima y enfatizar que tiene "aguante" para las peleas y adversidades.

La elección mayoritaria de ejemplos en los que participan mujeres ha sido deliberada. Un fenómeno emergente desde la perspectiva de género ${ }^{[s]}$ es que las mujeres despliegan performances de "aguante" que, según un sistema sexo-generizado y heteronormativo ${ }^{(42)}$, se asocian tradicionalmente a la masculinidad. Pensar esta dinámica en sectores populares desde una perspectiva de género visibiliza una femineidad no hegemónica vinculada al "aguante" en términos corporales y territoriales, y evidencia otra de las modulaciones posibles de un nuevo "tiempo de chicas"(29).

No obstante, como también se observa en otros trabajos ${ }^{(43)}$, estas modulaciones emergentes conviven en tensión con prácticas de masculinidades tradicionales. En uno de los 
ejemplos de portada analizados se podía observar la imagen que elegía un adolescente (17 años) para presentarse ante los otros: dos armas, la expresión "TE $A M O^{\prime}$ escrita con balas y abajo, entre dos cajas repletas de municiones, la frase "Pero si me fallas todas son para ty". Su aparente declaración de amor opera como una performance de amenaza a su pareja: el amor solo resultará posible si ella acepta la dinámica de poder y violencia propuesta. El efecto de amenaza se completa porque anula la posibilidad de negociación y establece que solo el varón tiene la potestad de leer la traición. La amenaza también se dirige a sus pares varones porque funciona como modo de legitimación del "aguante" entendido en una concepción sexo-generizada tradicional, en el sentido de las relaciones asimétricas de poder que los varones establecen con las mujeres.

Varios eligen esas fotos de portada
porque creen que vende mostrarse
celosos y guardianes. Se zarpan aunque
a algunas les re va eso. Muchas que-
remos un novio que sea cuida, fiel y re
leal pero los pibes se pasan de rosca y
a veces se drogan o toman demasiado
y se ponen violentos, no da. (Entrevista,
mujer, 18 años)

Los tres ejemplos seleccionados resultan en su conjunto paradigmáticos para analizar las expresiones de amenazas y la producción de subjetividad en las y los adolescentes de sectores populares marginalizados del AMBA. De este modo, coexisten órdenes de géneros tradicionales y emergentes. En el último ejemplo, se observa la persistencia, e incluso el peligro, de un orden de género tradicional que vincula la amenaza y el "aguante" a una masculinidad hegemónica en la que prima el real o potencial uso de la fuerza física, el control y el abuso de poder. Sin embargo, en los dos ejemplos previos se había mostrado la posibilidad de un orden de género emergente que amplía el valor del "aguante" para las mujeres, lo que posibilita que ellas exploren prácticas de femineidad no hegemónica.

\section{"Bondis"}

La categoría nativa que utilizan las y los adolescentes de sectores populares marginalizados del AMBA para referirse a las peleas es "bondi". No se trata simplemente de un sinónimo de "pelea", dado que implica un desplazamiento y una construcción en torno a este significante. ¿Qué se disputa en un "bondi"? ¿Cuáles son las estrategias desplegadas por sus participantes? A diferencia de las amenazas, los "bondis" suelen realizarse concretamente entre determinados usuarios.

Por su propia composición, el "bondi" también entrelaza prácticas offline y online. En numerosas ocasiones, el "bondi" comienza online, se viraliza entre la pequeña comunidad de pares y se proyecta hacia ámbitos copresenciales como el barrio, la escuela o la discoteca. En otras, el "bondi" se inicia en espacios offline y luego continúa de modo online, amplificando el problema inicial y sumando nuevos usuarios que se sienten interpelados y se involucran en la disputa.

Esto va para todas las gilas de pola! Yo no me voy andar rebajando ni de honda con ustedes, porque no se dejan de joder y de ultima si son tan buenas se paran de mano solas? Qe tienen qe andar metiendo alos familiares? Si ustedes mismas se buscan el bondi! Ya estan grandes loco ! Tienen hijos y siguen actuando como unas pendejas! Si derrepente papu sabe a qien qeria y a qien no ! Corta. No tienen qe andar haciendo cartel de nada \& qe les qede claro si tienen qe hacer algo lo hacen y fue ! Basta de hacer cartel por facebook , toi rre cachuda y si siguen hablando gilada voi aggarrar una por una! Yo hoy en dia no estoy para andar soportando a ninguna gila! Y mas vale qe lo llore porqe yo se qe el ami me queria! Y yo tambien. Nosvemos! No tengo porqe andar hablando por facebook nv.

(Publicación en Facebook, usuaria del AMBA) 
La transcripción anterior corresponde a la publicación de una adolescente (17 años) que da cuenta del "bondi" a raíz de un conflicto en torno al final de una relación amorosa y a las habladurías de mujeres cercanas.

\begin{abstract}
$A$ veces se arman bondis por Face. Por ahí arrancan en el colegio, siguen en Face y terminan en la calle. Muchas mujeres se agarran a la salida del colegio por cosas de Face. Chismes de que una estuvo con el novio de la otra, ponele. (Entrevista, mujer, 16 años)
\end{abstract}

Face está lleno de pibes y pibas pasando chismes y bardeos. Arman bondi por cualquier cosa, si uno estuvo con otro, si le dijo tal o cual cosa, si lo miró, si salió, se peleó. (Entrevista, varón, 17 años)

En los fragmentos de entrevistas, los adolescentes mencionan los problemas que surgen en torno a "chismes" y "bardeos". Estos conflictos se asocian al monitoreo que permite, y de algún modo estimula, la red social Facebook, que propone un seguimiento constante de las actividades de los contactos. Asimismo, puede pensarse que los frecuentes "bondis" también funcionan como medio para disputar el capital del "aguante" dado que, en estos conflictos, se pone a prueba el prestigio y el honor de cada participante en relación con las performances que realizan en torno a su capacidad de resistencia y osadía.

-ALTO, booonddy se armo con los pibes de mi barrio hace rato $X D$ ( LOS DE LA INTA, CONTRA LA 20) Que giles que son wacho -.- MAÑANA COBRAAN \& LA WACHA QUE SALTE COBRA TAMBIEN ah. DE UNA, MAÑANA CON LAS PIBAS A FUUUUL - NOS RE PARAMOS DE MANO CON QUIEN SEA, \& QUIEN SEA META en eL bondy. [emoticón] ;)) ᄀ

Encima estoy re caliente, con unas wachas que se hacias las piolas, JAJAJAJAJA XD como tocaron pa su barrio, CAGONAS, son re Gilas, no

\author{
se La bancaan que salen corriendo \\ [emoticón] \\ (Publicación en Facebook, usuaria del \\ AMBA)
}

En esta transcripción una adolescente (16 años) menciona que se armó "alto booondy" entre los pibes de dos barrios ("los de la Inta contra la 20"). También advierte que "mañana cobran \& la wacha que salte cobra también". Por último, insinúa que tanto ella como los de su barrio son quienes más "aguante" tienen y que los otros no se la "bancan" y siempre "salen corriendo". Este tipo de publicaciones sugiere un emergente orden de género en el que las mujeres de sectores populares marginalizados también tienen la posibilidad de pelear y tener "aguante". Desde el propio sentido que le otorgan, esto implica la legitimación subjetiva de poner el cuerpo para defender el honor y el territorio. La publicación recibió 30 "me gusta" y decenas de comentarios.

\section{Cyberbullying}

El bullying, concepto que proviene del inglés, refiere al acoso por parte de compañeros/as o pares a una sola persona. En la agenda mediática el término tuvo mucha pregnancia $y$, en especial, se lo asocia a la adolescencia y al contexto escolar. Incluye cualquier acción o comunicación hostil que un grupo de adolescentes realiza con otro: desde golpearlo hasta amenazarlo, sustraerle pertenencias, escribirle mensajes agresivos o no dirigirle la palabra ${ }^{(44)}$. En la era de los sitios de redes sociales, se popularizó el término cyberbullying, también denominado "ciberacoso" o "acoso digital", que refiere al bullying efectuado de modo virtual. Según Willard(44), el cyberbullying puede definirse brevemente como el envío o posteo vía Internet o telefonía móvil de contenidos (textos o imágenes) perjudiciales de modo intencionado hacia otro. Mason ${ }^{(45)}$ agrega que el cyberbullying es realizado por un solo individuo o un grupo, en ambos casos de modo deliberado y repetitivo. Es decir, 
por cyberbullying se entiende el envío de mensajes agresivos, las publicaciones que divulgan rumores o la decisión grupal de no dirigir ninguna acción hacia ese perfil. En numerosas ocasiones, tanto el bullying como el cyberbullying deben interpretarse como dos manifestaciones de un mismo conflicto que se retroalimentan mutuamente. Si bien existen distintas definiciones de estos fenómenos dentro de la bibliografía, existe consenso en que implican agresión, repetición y desigualdad de poder ${ }^{(44)}$. La ubicuidad y capacidad de las TIC para llegar a audiencias significativamente mayores es una característica específica del cyberbullying en comparación con otro tipo de $\operatorname{acoso}^{(20)}$. En esta línea, Kowalski, Limber y Agatston $^{(46)}$ sostienen que, según los adolescentes consultados, el anonimato tiende a facilitar el cyberbullying, ya que el acosador cuenta con mayores posibilidades de permanecer impune.

De este modo, a las expresiones de violencia que un grupo de usuarios despliega ante un solo usuario de la red aquí se propone agruparlas en torno al concepto ya explicado de cyberbullying. Se señala que, en este caso, a diferencia de la palabra nativa "bondi", se utiliza un término instalado por los medios masivos de comunicación y en idioma inglés, con las diferencias que esto supone respecto de la denominación anterior. Cabe aclarar, además, que esta problemática no es exclusiva de los adolescentes ni de los sectores populares marginalizados, dado que también se observa en otros sectores sociales y en otras franjas etarias, aunque sea más frecuente encontrar casos de cyberbullying a nivel internacional entre adolescentes ${ }^{(21,47,48)}$.

NO LA VOI A CAJETIAR NI DE ONDA CON LO QUE ME PUSIERON EN LAS PAREDES DEL COLEGIO DE MI , PERO Si TAN BUUEENAS SON PORQE SE QE FUE UNA ENVIDIOOOSA DE MIERDA POOOORQE NO PONEEEEEN NOOMBREEEEES ? PORQE SOOON RE CAGOOONAS PORQE SAAABEN QE YOO ME VOOOi A RE PARAR DE MAAAAAAAANO QE NO ME VOi A CAGAR CON NADIE ASI GANE O
PIRDA ME CHUPAN LA CAIETA YO PELEO \& NADA MAS! MANGAS DE GILAS PONGANLE NOMBRES (; SE CREEN PIOLAS? AJAJAJJAAJJAJAJAJAJAJA MUCHA LANA LORITAS PENGUENSE UN BAÑO \& DESPUES ABLAMOS NO ME LLEGAN NI A LOS TALONES XQ ASER ESO ES DE UNA WACHA ENVIDIOSA [emoticón] CORTiiTA MA [emoticón] (Publicación en Facebook, usuaria del AMBA)

En esta transcripción se observa la denuncia de bullying que realiza una adolescente (16 años) sobre los graffitis que aparecieron con su nombre en las paredes del colegio al que concurre. Al igual que numerosas publicaciones observadas en los muros de Facebook, estos graffitis actualizan un orden de género tradicional ante las prácticas sexoafectivas: los varones obtienen legitimidad social si mantienen y acumulan variadas experiencias mientras que, para las mujeres, en muchas ocasiones, la misma dinámica es cuestionada, incluso por sus pares mujeres. Además, aquí se observa otra modulación posible de las expresiones en torno a la violencia: una adolescente padece bullying copresencial y encuentra en la plataforma Facebook, es decir de modo online, la posibilidad de mostrar la violencia sufrida.

Otra práctica asociada al cyberbullying y observada con cierta recurrencia entre las y los adolescentes de la muestra es la que denominan "escrache". Originalmente, este término proviene del uso de agrupaciones de derechos humanos como Hijos e Hijas por la Identidad y la Justicia contra el Olvido y el Silencio (HIJOS), que "escrachaban" (señalaban, marcaban) a represores de la última dictadura militar que se encontraban sin condena efectiva. A partir de la masificación de este uso, la palabra fue apropiada por numerosos adolescentes del AMBA con sentidos particulares. Un sentido utilizado con frecuencia es el de una "foto escrache", en alusión a una selfie (autofoto) o foto personal tomada por otro que perjudica la imagen del usuario que aparece en la foto. Además de esta acepción, 
se presenta otra relacionada con la agresión o denuncia a través de Facebook.

En este contexto, el "escrache" es un recurso que sirve para alertar al grupo de pares sobre ciertas conductas violentas que realiza un usuario. Así, también funciona como cierto modo de empoderamiento que realizan algunas adolescentes de sectores populares marginalizados. Este tipo de advertencias circulan en ocasiones por la red de contactos: "cuidado con $[\mathrm{X}]$ que te cornea y no le importa nada salvo seducir por chat a cualquiera" (mujer, 17 años). "Me enamoré pero fui una ingenua. $[\mathrm{X}]$ me mintió y me la creí. Pero es solo chamuyo" (mujer, 16 años). Como se observa en numerosos ejemplos, esta denuncia de "escrache" hacia un varón suele tener un componente de duelo sexoafectivo, pero también de concientización de alertar al grupo de mujeres de su red de contactos acerca de las conductas sexo-genéricas de los varones.

Como se adelantó, la práctica del "escrache" también tiene una vertiente estética cuando una foto es tomada por alguien sin permiso del fotografiado. Esta expresión busca deslegitimar o avergonzar a quien aparece en la foto $y$, al mismo tiempo, aumentar la popularidad del autor de la publicación. Desde su origen, el "escrache" ha cambiado en sus modulaciones: ha pasado de ser una práctica grupal, con un fuerte anclaje sociopolítico de denuncia de crímenes de lesa humanidad, para transformarse en una práctica con componentes identitarios, estéticos y de "aguante" anclados al barrio, que mediante la plataforma se socializa con suma rapidez.

\section{Duelos}

Las y los adolescentes de sectores populares marginalizados experimentan con frecuencia la muerte de sus amigos, pares o conocidos, en ocasiones como consecuencia de episodios violentos ${ }^{(9,10)}$. En paralelo a estos sucesos, las sociedades vienen experimentando una transformación en los modos de elaboración de los duelos. Los ritos mortuorios se han modificado en las últimas décadas y el impacto de las TIC ha posibilitado el surgimiento de numerosas prácticas que implican el recordatorio de la persona fallecida en sitios de redes sociales. Las y los adolescentes de la muestra se reapropian de estas prácticas y las resignifican en su contexto específico, aunque también se observen componentes comunes con las acciones online que realizan algunos adultos, con la función de preservar la memoria de un contacto fallecido ${ }^{(49)}$.

En ciertos casos relevados, el perfil del adolescente fallecido es mantenido por íntimos o familiares. También estas prácticas unen al grupo ante la otredad: otros grupos de pares, bandas delictivas locales o la policía. Este tipo de publicaciones suelen estar marcadas por la tristeza y el deseo de justicia frente a los episodios violentos que tuvieron como consecuencia el fallecimiento del ser querido.

\begin{abstract}
A un ex compañero de la escuela lo mataron en un enfrentamiento entre bandas. Me hubiese gustado poder ser amiga de él. Ahora a veces lo extraño. Es raro que ya no esté. Hace poco publiqué algo en Face para recordarlo y apoyar a la familia y a los amigos, que están sufriendo bastante. Convivimos con estas cosas todo el tiempo. (Entrevista, mujer, 15 años)
\end{abstract}

En una de las imágenes analizadas, un adolescente (14 años) publica un fotomontaje en el que la cara de su hermano está superpuesta en transparencia sobre el cielo, y señala "Hoy Mi Corazon Solo Piesa En Ti, Hoy se Cumplen 1 año De q Ya No Estas AK y No Tenes iDea Lo Mucho q Te Extraño HermaniTo". En otra publicación similar, un adolescente (15 años) cuenta que con el compañero fallecido se sentaban juntos en la escuela primaria, que le hubiese gustado desarrollar una amistad y que no puede creer lo que le ocurrió. Las publicaciones tuvieron 65 y 92 "me gusta", respectivamente, señal del alto grado de aceptación de este tipo de recordatorios entre la red de contactos. 
Estos hechos traumáticos suelen ser incorporados a la configuración de los perfiles de adolescentes de sectores populares marginalizados. Incluso también forman parte de sus recaudos ante búsquedas sexo-afectivas, dado que perciben como traición o falta de "aguante" abandonar el duelo por el fallecido y no mencionarlo periódicamente en su biografía. En ocasiones, se agrega en la portada o en el nombre de perfil un recordatorio ("por siempre Maxi"; "te extraño Ranchi"). Contactos menos cercanos al faIlecido suelen dar expresiones de "me gusta" y dejar comentarios como gestos de apoyo a los allegados. Además, es usual encontrar reivindicaciones y pedidos de justicia por adolescentes fallecidos en situaciones no esclarecidas (hechos delictivos, "ajustes de cuentas", "gatillo fácil").

Hace unos años murió uno de mis mejores amigos. El barrio es inseguro, pero nos cuidamos entre todos. Cada dos por tres la policía nos revisa y a veces nos pega porque cree que hicimos algo malo solo por ser del barrio, o por bronca nomás, no sé. [...] A veces compartimos cosas en Face sobre él para recordarlo y para acompañar a la familia y a los amigos que están mal. (Entrevista, mujer, 16 años)

En estas prácticas confluyen publicaciones atravesadas por la amistad, el afecto y el duelo, como una nueva forma de transitar la muerte de los seres queridos. Facebook les provee un espacio donde pueden expresarse y recibir el feedback de otros pares, generando una interacción y, en ocasiones, la creación de un nuevo vínculo o el reforzamiento de uno anterior, que puede transformarse de diversas formas. A la vez, resulta preciso indagar aún más en este tipo de fenómenos para comprender cómo estos adolescentes resignifican sus subjetividades y los efectos de la violencia que padecen.

\section{DISCUSIÓN}

Dentro de las investigaciones que estudian a estas poblaciones -definidas aquí como adolescentes de sectores populares marginalizados- se retoman en particular los trabajos que analizan la violencia urbana. En este sentido, se propone como una contribución específica vincular estos aportes con el impacto de las TIC y, en especial, con el auge de los sitios de redes sociales. De las múltiples expresiones de violencia relevadas a través de la estrategia metodológica, el presente artículo se circunscribe a las exploradas en la red social Facebook: amenazas, "bondis", cyberbullying y duelos.

La revisión bibliográfica que realizan Valdivia y González ${ }^{(18)}$ evidencia que las amenazas entre jóvenes son frecuentes a nivel mundial y que las agresiones relacionales sexo-afectivas ocurren hasta en dos tercios de muestras de jóvenes de distintas latitudes. Como señalan estos investigadores, la mayoría de los estudios que abordan esta problemática lo hacen con jóvenes universitarios, cuya accesibilidad es mayor dado que suelen transitar las mismas instituciones que los docentes-investigadores. En este sentido, el presente artículo pretende aportar al campo la exploración de las modulaciones de las agresiones relacionales online entre adolescentes de sectores marginalizados.

Buelga y Pons $^{(22)}$ señalan que la frecuencia del cyberbullying entre los jóvenes varía, según distintos estudios mundiales, entre el $5 \%$ y el $34 \%$ y tal como sugieren los autores, la variabilidad estadística en las distintas investigaciones torna dificultosa la comparación. Esto se debe, en parte, a que no existe acuerdo sobre la definición de cyberbullying, ni tampoco sobre las variables que deben medirse ${ }^{(20,48)}$. En definitiva, aun con estas limitaciones, resulta imperioso continuar investigando la problemática del cyberbullying en Facebook dado que, según diversos estudios ${ }^{(24,34)}$, es la plataforma más utilizada por las y los adolescentes para realizar cyberbullying y otras expresiones de violencia online. 
Los estudios pioneros sobre expresiones de violencia online entre adolescentes se desarrollaron en gran medida en Europa Occidental, EE.UU. y Australia(20,45,46,48,50). Estas investigaciones han sido las primeras en abordar estos fenómenos -en particular, el cyberbullying- porque las TIC tendieron a masificarse en estos países más rápidamente que en los demás. No obstante, la bibliografía reciente señala que, aunque los fenómenos de violencia digital entre adolescentes continúan siendo frecuentes en esas áreas geográficas $^{(22,47,48)}$, también se desarrollan progresivamente en Latinoamérica ${ }^{(23,24)}$. En este sentido, dada la escasez de estudios empíricos a nivel latinoamericano ${ }^{(23,24)}$, y en particular entre adolescentes de sectores populares, resulta necesario profundizar las investigaciones, a la luz de las características específicas de las sociedades y economías latinoamericanas.

¿Por qué Facebook, la red icónica del "me gusta", habilita también expresiones de violencia? ¿De qué modos las y los adolescentes de sectores populares marginalizados producen estas expresiones o las resignifican en sus contextos específicos? ¿Cuáles son las distintas modulaciones que adquieren las expresiones de violencia en mujeres y varones?

Fraternidad, liderazgo, protección, cohesión grupal y legalidades propias constituyen, así, marcas de un "nosotros juvenil" donde eventualmente se incluyen actos violentos cuyos significados deben inscribirse en el contexto de condiciones sociales disponibles. Frente a tal panorama se dibujan varias opciones: desoír ciertos modos de presentación de la condición juvenil; expulsarlos; percibirlos como fatalidad; o preguntarnos qué puede verse allí más allá de la violencia. ${ }^{19}$ p.354)

La pregunta de investigación de este artículo se construye en la intersección de varias discusiones contemporáneas en las ciencias sociales. En especial, se retoman los trabajos de Auyero et al. ${ }^{(14)}$, quienes describen las múltiples formas de violencia copresencial que atraviesan los adolescentes de sectores socialmente vulnerables del AMBA. Estas expresiones pueden observarse, como señalan los autores, en los graffitis de las paredes de los barrios. De manera análoga, en los muros de Facebook son frecuentes las publicaciones de recordatorios visuales de las consecuencias de la violencia. En línea con Duschatsky ${ }^{(19)}$, lo que se propone en este artículo no es simplemente catalogar conductas disruptivas, sino explorar distintas formas en las que estos adolescentes procuran otorgarle sentidos a sus prácticas cotidianas. Queda pendiente para futuras investigaciones continuar indagando en qué medida las herramientas tecnológicas proyectan las prácticas violentas -inscriptas en el más amplio campo de los fenómenos de violencia urbana- o también aumentan las "condiciones sociales disponibles"(19) y contribuyen al empoderamiento de las y los adolescentes de sectores populares marginalizados para contextualizar, resignificar o desplazar los sentidos sociales hegemónicos atribuidos a la violencia.

\section{CONCLUSIONES}

Este artículo ha indagado, mediante una metodología mixta que incluye una etnografía digital, las expresiones de violencia que realizan las y los adolescentes de sectores populares marginalizados del AMBA en los muros de Facebook. De este modo, se evidencia que las redes online se encuentran insertas en su entramado social, y que este tipo de etnografía resulta una herramienta innovadora para explorar sus prácticas. La mayoría de las interacciones que incluyen violencia se desarrollan de modo offline-online, dado que numerosos conflictos comienzan en el plano offline (por ejemplo, en el colegio o en una plaza) y continúan en la plataforma virtual, o viceversa.

En este sentido, se han analizado cuatro formas de violencia en la red social Facebook: amenazas, "bondis", cyberbullying y duelos. En primer lugar, las expresiones de amenaza, descriptas como modo de advertencia de 
una violencia futura, dan cuenta de ciertas tensiones en torno al territorio, el honor, el respeto y los vínculos sexo-afectivos. En segundo lugar, los "bondis", categoría nativa que alude a peleas, evidencia no solo un continuum de relaciones offline-online, sino también la centralidad que ha adquirido el plano virtual como nueva arena de enfrentamientos. En tercer lugar, el cyberbullying, en tanto acciones online agresivas sobre un único usuario, enfatiza que el acoso u hostigamiento encuentra en Facebook un canal específico para desplegarse. En cuarto lugar, las expresiones de duelo que estos adolescentes realizan en Facebook representan un fenómeno característico de este sector social y permiten explorar cómo resignifican las distintas dimensiones de la violencia que los atraviesan.

Las cuatro formas se encuentran asociadas al "aguante", fenómeno sociocultural cuyos sentidos, en estos casos, refieren a "bancar", "defender" y "resistir". Una de las dinámicas exploratorias advertidas $-\mathrm{y}$ que merece ser objeto de futuras investigacioneses la emergencia de prácticas de "aguante" en mujeres. Estas expresiones implican nuevos órdenes de género, aunque éstos aún convivan con la persistencia de masculinidades y femineidades tradicionales, que también han sido relevadas. Este fenómeno específico de sectores populares señala la complejidad que posee la cultura del "aguante" en la intersección de la producción de subjetividad contemporánea masculina y femenina.

Finalmente, el artículo se pregunta en qué medida las expresiones abordadas resultan implícitamente funcionales a la red social o, por el contrario, permiten reapropiaciones significativas de los usuarios. Si se considera el "me gusta" como una moneda de cambio intrínseca de la red social que fortalece la adhesión al grupo de pares, estas expresiones revelan prácticas de ritualización grupal que, más allá de ser leídas como violentas, deben abordarse de manera contextualizada. Según la Real Academia Española, "violento" es aquel que "está fuera de su natural estado, situación o modo" y aquello "que se ejecuta contra el modo regular o fuera de razón y justicia". Cabe pensar si las expresiones de violencia de estos adolescentes están "por fuera" de su "estado, razón y justicia", o por fuera de otro "estado, razón y justicia" que aún no termina de integrarlos sino que, en numerosas ocasiones, solo los visibiliza con el estigma de "violentos".

\section{REFERENCIAS BIBLIOGRÁFICAS}

1. Foucault M. Vigilar y castigar. Buenos Aires: Siglo XXI Editores; 2002.

2. Foucault M. Historia de la sexualidad I. Buenos Aires: Siglo XXI Editores; 2002.

3. Benjamin W. Para una crítica de la violencia y otros ensayos. Madrid: Santillana; 1991.
4. Sloterdijk P. Normas para el parque humano: Una respuesta a la carta sobre el humanismo. Madrid: Siruela; 2000.

5. Rancière J. El desacuerdo: Política y filosofía. Buenos Aires: Nueva Visión; 1996.

6. Isla A, Míguez D. Formations of violence in post-dictatorial contexts: logics of confrontation between the police and the young urban poor in 
contemporary Argentina. International Journal of Conflict and Violence. 2011;5(2):240-260.

7. Spinelli $\mathrm{H}$, Alazraqui $M$, Macias G, Zunino M, Nadalich J. Muertes violentas en la Ciudad Autónoma de Buenos Aires: una mirada desde el sector salud. Buenos Aires: OPS; 2005.

8. Tonkonoff S, (ed). Violencia y cultura: Reflexiones contemporáneas sobre Argentina. Buenos Aires: Clacso; 2014.

9. Imbusch P, Misse M, Carrión M. Violence research in Latin America and the Caribbean: A literature review. International Journal of Conflict and Violence. 2011;5(1):87-154.

10. Auyero J, Berti M. La violencia en los márgenes. Buenos Aires: Katz; 2013.

11. Kessler G, Dimarco S. Jóvenes, policía y estigmatización territorial en la periferia de Buenos Aires. Espacio Abierto. 2013;22(2):221-243.

12. Instituto Nacional de Estadística y Censos. Censo Nacional de Población, Hogares y Viviendas 2010. Buenos Aires: INDEC; 2010.

13. Wacquant L. Los condenados de la ciudad. Buenos Aires: Siglo XXI Editores; 2007.

14. Auyero J, Burbano de Lara A, Berti M. Uses and forms of violence among the urban poor. Journal of Latin American Studies. 2014;46(3):443-469.

15. Di Leo P. Cuerpos, vulnerabilidades y reconocimiento: las violencias en las experiencias y sociabilidades juveniles. En: Di Leo P, Camarotti A. 'Quiero escribir mi historia': Vidas de jóvenes en barrios populares. Buenos Aires: Biblos; 2013.

16. Morduchowicz R. Los adolescentes y las redes sociales. Buenos Aires: FCE; 2012.

17. Misse M. La acumulación social de la violencia en Río de Janeiro y en Brasil: Algunas reflexiones. Co-Herencia. 2010;7(13):1-19.

18. Valdivia M, González L. Violencia en el noviazgo y pololeo: una actualización proyectada hacia la adolescencia. Revista de Psicología. 2014;32(2):330-355.

19. Duschatsky S. Veo veo... ¿Qué ves?: Percepciones más allá (o más acá) de la violencia. Revista CS. 2011;2:343-366.

20. Smith P, Mahdavi J, Carvalho M, Fisher S, Russell S, Tippett N. Cyberbullying: its nature and impact in secondary school pupils. Journal of Child Psychology and Psychiatry. 2008;49(4):376-385.
21. Del Río Pérez J, Sádaba Chalezquer Ch, Bringué Sala X. Menores y redes isociales?: de la amistad al cyberbullying. Revista de Estudios de Juventud. 2010;(88):115-129.

22. Buelga S, Pons J. Agresiones entre adolescentes a través del teléfono móvil y de Internet. Psychosocial Intervention. 2012;21(1):91-101.

23. Wendt G, Lisboa C. Agressão entre pares no espaço virtual: definições, impactos e desafios do cyberbullying. Psicologia Clínica. 2013;25(1):73-87.

24. Menay-López L, de la Fuente-Mella H. Plataformas comunicacionales del ciberbullying: Una aplicación empírica en dos colegios de la quinta región, Chile. Estudios Pedagógicos. 2014;XL(2):117-133.

25. Aisenson D, Batlle S, Aisenson G, Legaspi L, Vidondo M, Nicotra D, Valenzuela V, Davidzon S, Ruiz de Isaac A, Polastri G, Alonso D. Desarrollo identitario de los jóvenes y contextos significativos: una perspectiva desde la psicología de la orientación. Anuario de Investigaciones. 2006;13:81-88.

26. Krauskopf D. Adolescencia y educación. San José: Euned; 2007.

27. Linne J. Autopresentación, amistad y vínculos sexo-afectivos en sitios de redes sociales en adolescentes de sectores populares de la Ciudad de Buenos Aires. [Tesis de doctorado]. Universidad de Buenos Aires, Facultad de Ciencias Sociales; 2015.

28. Urresti M, Linne J, Basile D. Conexión total: Los jóvenes y la experiencia social en la era de la comunicación digital. Buenos Aires: Grupo Editor Universitario; 2015.

29. Elizalde S. Tiempo de chicas: Identidad, cultura y poder. Buenos Aires: Grupo Editor Universitario; 2015.

30. Margulis $M$, Urresti $M$, Lewin $H$. Familia, hábitat y sexualidad en Buenos Aires. Buenos Aires: Biblos; 2007.

31. Administración Nacional de la Seguridad Social. Conectar Igualdad [Internet]. Buenos Aires: Presidencia de la Nación; 2013 [citado 2 dic 2015]. Disponible en: http://goo.gl/mKiayJ.

32. Ministerio de Educación. Plan de Finalización de Estudios Primarios y Secundarios - FINES [Internet]. Buenos Aires: Presidencia de la Nación; 2009 [citado 2 dic 2015]. http://goo.gl/f7AJZI. 
33. Hine C. The Internet: understanding qualitative research. New York: Oxford University Press; 2012.

34. Boyd D. It's complicated: the social lives of networked teens. New Haven: Yale University Press; 2014

35. Alabarces $P$, Rodríguez $M$, (org). Resistencias y mediaciones: estudios sobre cultura popular. Buenos Aires: Paidós; 2008.

36. Garriga Zucal J. 'Soy macho porque me la aguanto': Etnografía de las prácticas violentas y la conformación de identidades de género masculino. En: Alabarces P, et al. Hinchadas. Buenos Aires: Prometeo; 2005.

37. Urresti M. De la cultura del aguante a la cultura del reviente: cambios en la significación de la corporalidad en adolescentes y jóvenes de sectores populares. En: Margulis M, Urresti M, Lewin $\mathrm{H}$. Familia, hábitat y sexualidad en Buenos Aires. Buenos Aires: Biblos; 2007.

38. Míguez D. Estilos musicales y estamentos sociales: Cumbia, villa y transgresión en la periferia de Buenos Aires. En: Míguez D, Semán P. Entre santos, cumbias y piquetes. Buenos Aires: Biblos; 2006.

39. Semán P, Vila P. Cumbia villera: una narrativa de mujeres activadas. En: Semán P, Vila P. Cumbia: Nación y género en Latinoamérica. Buenos Aires: Gorla; 2011.

40. Silba M. Vidas plebeyas: masculinidades, resistencias y aguante entre varones jóvenes pobres del Conurbano. Papeles de Trabajo. 2012;6(10):160-176.

41. Rubin G. The traffic in women: Notes on the 'political economy' of sex. In: Reiter R, (ed). Toward an anthropology of women. New York: Monthly Review Press; 1975.

42. Butler J. El género en disputa: El feminismo y la subversión de la identidad. Barcelona: Paidós; 2007.

43. Linne J. Usos comunes de Facebook en adolescentes de distintos sectores sociales. Comunicar. 2014; XII(43):189-197.

44. Willard N. An educator's guide to cyberbuIlying and cyberthreats [Internet]. 2005 [citado 12 dic 2015]. Disponible en: https://goo.gl//4TM4H.

45. Mason K. Cyberbullying: A preliminary assessment for school personnel. Psychology in the School. 2008;45(4):323-348.
46. Kowalski R, Limber S, Agatston P. Cyber buIlying: Bullying in the digital age. London: Blackwell Publishing; 2008.

47. Garaigordobil M. Prevalencia y consecuencias del cyberbullying: una revisión. International Journal of Psychology and Psychological Therapy. 2011;11(2):233-254.

48. Bottino S, Bottino C, Cássio R, Regina C, Ribeiro W. Cyberbullying and adolescent mental health: systematic review. Cadernos de Saúde Pública. 2015;31(3):463-475.

49. Ramos H. Além-túmulo no Facebook: Vida após a morte e luto na Era Digital. Observatorio (OBS*). 2015;9(4):31-50.

50. Price $M$, Dalgleish J. Cyberbullying: Experiences, impacts and coping strategies as described by Australian young people. Youth Studies Australia. 2010;29(2):51-59.

\section{NOTAS FINALES}

a. Por AMBA se alude a la Ciudad de Buenos Aires y al denominado Gran Buenos Aires, que incluye los Municipios de Almirante Brown, Avellaneda, Berisso, Berazategui, Cañuelas, Ensenada, Escobar, Esteban Echeverría, Ezeiza, Florencio Varela, General Rodríguez, General San Martín, Hurlingham, Ituzaingó, José C. Paz, La Matanza, La Plata, Lanús, Lomas de Zamora, Malvinas Argentinas, Marcos Paz, Merlo y Moreno. Según el Instituto Nacional de Estadística y Censos (INDEC), en el AMBA reside alrededor de un tercio de la población argentina.

b. El programa Conectar Igualdad fue una iniciativa del gobierno nacional argentino desde 2010 y consistió en entregar una netbook en comodato a cada alumno y docente de escuelas públicas secundarias del país. En el marco de esta política, algunos municipios del AMBA implementaron centros de inclusión digital en los cuales los jóvenes podían acceder al uso gratuito de computadoras con acceso a Internet, y contar con la orientación de técnicos y facilitadores docentes.

c. El Plan Fines fue una iniciativa del gobierno nacional argentino desde 2008 y consistió en la posibilidad de que adultos que no habían podido terminar el colegio secundario pudieran hacerlo en una modalidad de cursada más flexible ${ }^{(31,32)}$.

d. Por "bardeos" la población de estudio construye el significado de "generar conflictos" u "hostigar a otros", y por "pararse de manos" construye el significado de "defenderse o mostrarse sin miedo ante una eventual pelea". 
e. Por "arrugar" la población de estudio construye el significado de "carecer de aguante".

f. Algunas de las bandas de cumbia que aparecen mencionadas en publicaciones de Facebook de los adolescentes de la muestra son: Alto faso, Corre guachín, El borracho, El punga, Entre rejas, Flashito Tumbero, Flor de Piedra, Jala jala, La mafia, La rama, La tiza, La yerba del mono, Mala fama, Pala ancha, Pibes Chorros, Prende la vela, Sobredosis, Supermerka2, Tinta roja, Una de kal, Vagancia y Yerba Brava. g. Se aclara que la perspectiva de género aquí se aborda, principalmente, a partir de los conceptos "sistema sexo-género"(41), que refiere al conjunto de disposiciones por el que una sociedad transforma la sexualidad biológica en productos de la actividad humana, y de "performatividad"(39), que alude a que el sexo es una construcción tan cultural como el género y que la performance de género está atravesada por una matriz heteronormativa.

\section{FORMA DE CITAR}

Linne JW, Angilletta MF. Violencia en la red social: Una indagación de expresiones online en adolescentes de sectores populares marginalizados del Área Metropolitana de Buenos Aires. Salud Colectiva. 2016;12(2):279-294. doi: $10.18294 /$ sc.2016.741.

Recibido: 30 de septiembre de 2015 | Versión final: 23 de diciembre de 2015 | Aprobado: 18 de febrero de 2016

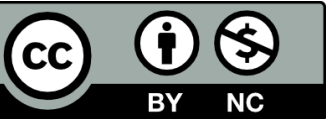

Este obra está bajo una licencia de Creative Commons Reconocimiento-NoComercial 4.0 Internacional. Reconocimiento - Permite copiar, distribuir y comunicar públicamente la obra. A cambio, se debe reconocer y citar al autor original. No Comercial - Esta obra no puede ser utilizada con finalidades comerciales, a menos que se obtenga el permiso.

http://dx.doi.org/10.18294/sc.2016.741 